\title{
A Comparison of Turkish-English Bilinguals' Processing of Emotion Words in Their Two Languages
}

\author{
Filiz Mergen ${ }^{a} *$ (D), Gulmira Kuruoglu b (D) \\ a İzmir University of Economics, No: 156, Balçova, İzmir, 35330, Turkey \\ ${ }^{b}$ Dokuz Eylul University, No: 144, Konak, 35220, Turkey
}

Received 22 May 2017 | Received in revised form 21 July 2017 | Accepted 12 September 2017

\begin{abstract}
There is a great deal of evidence showing that, in monolinguals, various emotional stimuli are processed by the brain in different ways. This view has found considerable support from studies conducted with verbal stimuli. In bilinguals, on the other hand, emotional processing is more complex, and is thought to be influenced mainly by two factors; age of language acquisition and proficiency. In this study, participants were forty-eight simultaneous / early bilinguals, who acquired both languages from birth, and have high proficiency in both. A lexical decision task, i.e., distinguishing real words from non-words, was used to gain insight into to how the participants processed visually presented emotion words in Turkish and English. Reaction times and accuracy were recorded via SuperLab software program and were statistically analyzed. Shorter response times and higher accuracy rates were found for real words compared to non-words in both languages. Also, shorter response times were found for positive compared to negative and neutral words in both languages. An analysis of the accuracy rates revealed no statistically significant differences among Turkish emotion words, whereas, for English, accuracy rates were higher for positive words when compared to negative and neutral words. These results have been interpreted in the light of psycholinguistic models of lexical processing
\end{abstract}

(C) 2017 EJAL \& the Authors. Published by Eurasian Journal of Applied Linguistics (EJAL). This is an open-access article distributed under the terms and conditions of the Creative Commons Attribution license (CC BY-NC-ND) (http://creativecommons.org/licenses/by-nc-nd/4.0/).

Keywords: Bilingualism, lexical processing, emotion words, lateralization

\section{Introduction}

It is well-established in the literature that emotional content of stimuli has a processing advantage, whether visual or auditory. Studies using verbal stimuli, mainly conducted with monolinguals, have provided supporting evidence (Kissler, Herbert, Winkler, \& Junghofer, 2009). It is not clear, however, whether this applies to the bilingual population. This uncertainty is attributed to the diversity of bilinguals' language experience, as confirmed by a majority of studies.

\footnotetext{
* Filiz Mergen.

E-mail address: filiz.mergen@ieu.edu.tr

http://dx.doi.org/...
} 
Bilingualism, characterized as being in a state of constant change depending on the frequency of use of each language, is a multi-faceted phenomenon. It is sufficiently common to be considered a norm, rather than an exception. In studies investigating bilingualism, the participants' age of acquisition, frequency of use and proficiency of languages are diverse. Particularly, the proportion of those acquiring their second language after their first is relatively high. Due to the diversity in participants' background, a variety of definitions of bilingualism has emerged. For example, based on the age of acquisition, bilinguals are labelled simultaneous, early or late bilinguals. They are categorized as proficient or non-proficient based on the proficiency level of both languages. Compound or Coordinate bilinguals are classifications according the cognitive structuring of the languages in the brain.

It is suggested that the diverse nature of bilingualism has implications for emotion word processing (Pavlenko, 2004). To start with, linguistic features of both languages should be considered, as well as structural and conceptual differences. There are some cases in which there is a complete overlap between concepts, while in others there may be striking differences (Pavlenko, 2008). Worldview of different cultures has a huge impact in the way emotion words are perceived. To be more specific, individualistic cultures and collectivist cultures differ in responses to the same emotions. For example, western cultures disapprove of dependence, while it is favored in collectivist cultures, such as Japan (Pavlenko, 2008).

Bilinguals have two languages at their disposal to express their emotions. A good deal of evidence has shown that different factors affect bilinguals' language preference in the expression and perception of emotional language, the most important being age of acquisition (Dewaele, 2004a; 2008; Harris, Ayçiçeği, \& Gleason, 2003; Harris, 2004). The prevalent view in the literature is that separate systems mediate language and emotions, and that these develop simultaneously early in infancy, which explains the more emotional nature of the native language (Pavlenko, 2004, 2012). Another view is that emotion words are more easily retrieved from the memory and more frequently used due to their richer mental representations originating with childhood experiences (Altarriba, 2006). This suggests that emotion words have deeper emotional associations (Dewaele, 2004a). Correspondingly, in the case of late acquisition of a second language, emotionality is perceived to be less, particularly if learned in formal settings (Dewaele, 2004a, 2004b; Pavlenko, 2004). However, L2 can sometimes be more emotional than the native language and preferred as the language to express emotions (Pavlenko, 2004, 2012).

Few studies investigated bilinguals' production and perception of emotionality. One possible reason is uncertainty over the validity of the results in the literature, due to "the diversity in the language experiences of the participants" (Harris, Gleason, \& Ayçiçeği, 2006, p. 258). This view is associated with the heterogeneous nature of bilingualism, revealed by conflicting results in the studies investigating emotionality in bilingual language production and perception. 
As confirmed by the majority of studies conducted with monolingual participants, compared to neutral words, emotion words are processed faster and more accurately, and recalled better (Nagae \& Moscovitch, 2002; Brierley et al., 2007). Bilingual literature has yielded similar results (Ferre, Garcia, Frage, Sanchez-Casas, \& Molero, 2010). However, there is no consensus regarding the emotionality of each language of bilinguals. For example, Anooshian and Hertel (1994) reported better recall of L1 emotion words compared to L2. Ferre et al., (2010), on the other hand, reported no difference in the recall of L1 and L2 words regardless of age and manner of acquisition, proficiency level or dominance of languages. Another line of research based on bilinguals' self-reports on their language choice to express emotions has provided much insight into the relationship between emotionality and the languages spoken.

Psycholinguistic research has shown that, like monolinguals, bilinguals process emotion words faster than neutral words. However, due to the diversity in the language experience of the participants, the majority of whom are late learners of L2, there have been doubts about the validity of the results obtained in bilinguality literature. In Eilola, Havelka and Sharma's study (2007), for example, no significant difference was found between the participants' languages in their processing of emotion words, which was interpreted as revealing equal perception of emotionality in both languages of proficient bilinguals. In contrast, Jonczyk (2013), reported shorter latencies and higher accuracy rates for negative words. This result was supported by another study conducted with late bilinguals (Jonczyk, 2014). Better performance in processing negative words were reported for Chinese-English bilinguals by Chen (2015). Late learners of L2 in Conrad, Recio and Jacobs' study (2011) processed emotion words in their L1 (German) and L2 (Spanish) faster and more accurately than neutral words. However, these results are contradicted. Early bilinguals in Altarriba and Basnight-Brown's (2010) study performed better in processing the emotion words in their L2 (English) than their L1 (Spanish). This result was associated with the frequency of use of their L2. L2 emotion words were found less effective than their L1 (English) by the late learners in Harris (2004), whereas no difference in emotionality was found between languages for early bilinguals. Sutton, Altarriba, Gianico, \& Basnight-Brown (2007) reported that L1 (Spanish) and L2 (English) were similar in terms of their emotionality for early bilinguals. In order to provide further support to bilinguality literature, this study aims to investigate perception of emotionality in simultaneous Turkish-English bilinguals.

\section{Method}

\subsection{Participants}

48 bilinguals who acquired Turkish and and English from birth participated in the study (15 Male, 33 Female, Mean Age= 29.75, $S t d=9.64$ ). A questionnaire was used to identify whether participants were eligible for the study. They were strongly righthanded (93.33\%, Std=10.49) as confirmed by the Edinburgh Handedness Inventory 
(Oldfield, 1971). The participants self-assessed their proficiency in both languages, and a Friedman Test revealed no significant difference between four language skills, $\mathrm{X}^{2}=5.21, s d=3, p=.157>.05$.

\subsection{Experiment}

Participants performed a lexical decision task in which they decided whether visually presented letter strings were real or non-words. They were instructed to perform the task as fast and accurately as possible. A trial session was conducted to familiarize the participants with the task.

The stimuli comprised of a total of 120 words and non-words. In the Turkish set, the real words were chosen from a pool of 300 words from Yazllı Türkçenin Kelime Sıllı̆̆l Sözlüğ̈̈ (Göz, 2003), and rated by a hundred Turkish native speakers on a 5point Likert Scale according to their valence, frequency of use and, the degree of arousal. After rating, 10 positive, 10 negative and 10 neutral words were selected from the pool. No significant differences were found in terms of frequency of use, $\left(F_{2,27}=0.83, p>.05, \mathrm{y}^{2}=.058\right)$. However, they differ significantly in terms of valence, $\left(F_{2,27}=98.01, p<.001, \mathrm{n}^{2}=.879\right)$. Non-words were created by exchanging the initial letters and final letters of real words, and they all complied with the phonotactic rules of Turkish. Similarly, the English set were formed from a pool of 300 words selected from Affective Norms for English Words (Bradley \& Lang, 1999). Based on the ratings collected from 30 English native speakers, 10 positive, 10 negative and 10 neutral words were selected for the English set. 30 non-words were formed by changing one letter of English real words.

The experiment took place in a quiet, dimly-lit room. The participants sat $40 \mathrm{~cm}$ away from a laptop computer using a chinrest. They were instructed to indicate whether the visually presented words were real words or non-words by pressing the designated keys on the keyboard ( 1 for yes, 2 for no), as required by the lexical decision task. Their response times and the accuracy of their answers were recorded via a software program, and SPSS was performed to analyze the data.

\section{Results}

Table 1 shows response times of the bilingual participants to emotion words in Turkish.

Table 1: Bilinguals' Response Times to Emotion Words in Turkish

\begin{tabular}{llllll} 
Turkish Words & Mean $(\mathrm{ms})$ & $\mathrm{N}$ & Std & $t$ & $p$ \\
\cline { 1 - 1 } Positive Words & 695.51 & 48 & 118.93 & -2.977 & $.005^{*}$ \\
\cline { 1 - 1 } Negative Words & 746.61 & & & \\
\hline Positive Words & 675.51 & 48 & 122.49 & -2.321 & .025 \\
\cline { 1 - 1 } Neutral Words & 736.55 & & & \\
\hline
\end{tabular}




\begin{tabular}{|c|c|c|c|c|c|}
\hline Positive Words & 695.51 & 48 & 143.06 & -9.019 & $.000 *$ \\
\hline Non-words & 881.73 & & & & \\
\hline Negative Words & 746.61 & 48 & 112.55 & .619 & .539 \\
\hline Neutral Words & 736.55 & & & & \\
\hline Negative Words & 746.61 & 48 & 129.89 & -7.207 & $.000 *$ \\
\hline Non-words & 881.73 & & & & \\
\hline Neutral Words & 736.55 & 48 & 142.12 & -7.077 & $.000^{*}$ \\
\hline Non-words & 881.73 & & & & \\
\hline
\end{tabular}

${ }^{*} p<.0083$ ( $p$ value has been adjusted by dividing .05 by the number of pair-wise comparisons, 6 in this case)

A paired-samples $t$-test revealed no difference in the response times for positive vs. neutral words and negative vs. neutral words, suggesting that, in terms of required processing times, emotion words were similar to neutral words. All three word types (positive, negative and neutral) were processed faster than nonwords. Also, positive words were processed faster than negative words.

A Wilcoxon test was performed on the accuracy data. Table 2 shows the differences in the accuracy rates of the Turkish words.

Table 1: Accuracy Rates for Emotion Words in Turkish

\begin{tabular}{|c|c|c|c|c|}
\hline Turkish Words & Mean (\%) & Std & $Z$ & $p$ \\
\hline Positive Words & 83 & .17 & -1.779 & .075 \\
\hline Negative Words & 78 & .16 & & \\
\hline Positive Words & 83 & .17 & -.369 & .712 \\
\hline Neutral Words & 82 & .15 & & \\
\hline Positive Words & 83 & .17 & -5.820 & $.000^{*}$ \\
\hline Non-words & 56 & .09 & & \\
\hline Negative Words & 78 & .16 & -1.176 & .240 \\
\hline Neutral Words & 82 & .15 & & \\
\hline Negative Words & 78 & .16 & -5.695 & $.000^{*}$ \\
\hline Non-words & 56 & .09 & & \\
\hline Neutral Words & 82 & .15 & -5.774 & $.000^{*}$ \\
\hline Non-words & 56 & .09 & & \\
\hline
\end{tabular}

${ }^{*} p<.0083$ ( $p$ value has been adjusted by dividing .05 by the number of pair-wise comparisons, 6 in this case)

Table 2 shows that positive, negative and neutral words were processed significantly more accurately than non-words. No other pair-wise comparisons in Table 2 were found significant.

Table 3 shows bilingual participants' response time to English words.

Table 3: Bilinguals' Response Times to Emotion Words in English. 


\begin{tabular}{|c|c|c|c|c|c|}
\hline Positive Words & 673.73 & 48 & 164.73 & -3.491 & $.001^{*}$ \\
\hline Negative Words & 756.75 & & & & \\
\hline Positive Words & 673.73 & 48 & 151.87 & -1.257 & .215 \\
\hline Neutral Words & 701.28 & & & & \\
\hline Positive Words & 673.73 & 48 & 169.05 & -7.645 & $.000^{*}$ \\
\hline Non-words & 860.28 & & & & \\
\hline Negative Words & 756.75 & 48 & 134.46 & 2.858 & $.006^{*}$ \\
\hline Neutral Words & 701.28 & & & & \\
\hline Negative Words & 756.75 & 48 & 155.67 & -4.608 & $.000^{*}$ \\
\hline Non-words & 860.28 & & & & \\
\hline Neutral Words & 701.28 & 48 & 137.27 & -8.025 & $.000^{*}$ \\
\hline Non-words & 860.28 & & & & \\
\hline
\end{tabular}

${ }^{*} p<.0083$ ( $p$ value has been adjusted by dividing .05 by the number of pair-wise comparisons, 6 in this case)

As can be seen in Table 3, response times for positive words are significantly shorter than those for negative words and non-words. However, no significant difference was found between positive and neutral words. Neutral words are processed significantly faster than negative words. Non-words yielded the longest response times when compared to positive, negative and neutral words, and the differences were found significant.

A Wilcoxon test was performed on the accuracy data, and the results are given in Table 4.

Table 2: Accuracy Rates for Emotion Words in English

\begin{tabular}{|c|c|c|c|c|}
\hline English Words & Mean (\%) & Std & $Z$ & $p$ \\
\hline Positive Words & 83 & .15 & \multirow[t]{2}{*}{-3.729} & \multirow[t]{2}{*}{$.000^{*}$} \\
\hline Negative Words & 73 & .15 & & \\
\hline Positive Words & 83 & .15 & \multirow[t]{2}{*}{-3.598} & \multirow[t]{2}{*}{$.000 *$} \\
\hline Neutral Words & 73 & .15 & & \\
\hline Positive Words & 83 & .15 & \multirow[t]{2}{*}{-6.032} & \multirow[t]{2}{*}{$.000^{*}$} \\
\hline Non-words & 58 & .06 & & \\
\hline Negative Words & 73 & .15 & \multirow[t]{2}{*}{-.056} & \multirow[t]{2}{*}{.955} \\
\hline Neutral Words & 73 & .15 & & \\
\hline Negative Words & 73 & .15 & \multirow[t]{2}{*}{-5.678} & \multirow[t]{2}{*}{$.000^{*}$} \\
\hline Non-words & 58 & .06 & & \\
\hline Neutral Words & 73 & .15 & \multirow[t]{2}{*}{-5.599} & \multirow[t]{2}{*}{$.000^{*}$} \\
\hline Non-words & 58 & .06 & & \\
\hline
\end{tabular}

${ }^{*} p<.0083$ ( $p$ value has been adjusted by dividing .05 by the number of pair-wise comparisons, 6 in this case)

According to Table 4, all pair-wise comparisons, except that between negative and neutral words are significant. Positive words have the highest accuracy rate when compared to negative words, neutral words and non-words. However, no significant difference was found between the negative and neutral words. 


\section{Discussion}

The aim of this study was to investigate emotion word processing in simultaneous bilinguals. An analysis of the RT's showed that positive words were processed faster than negative words both in Turkish and English. This result is in line with the majority of studies investigating emotion word processing, which highlight The Positivity Effect, i.e. the faster processing of positive words. This idea has an evolutionary basis, suggesting that the human brain prioritises positive stimuli to exploit the resources that are potentially advantageous for the organism in order to ensure its survival. This result has previously been reported in studies which employed verbal (Briesemeister, Kuchinke \& Jacobs, 2011; Kissler \& Koessler, 2011; Palazova, Mantwill, Sommer, \& Schacht 2011; Scott, O’Donnell, Leuthold, \& Sereno, 2009) and nonverbal stimuli (Schacht \& Sommer, 2009). This view has also been supported by electrophysiological (Kissler et al., 2009) and imaging studies (Kuchinke, Jacobs, Grubich, Vo, Conrad, \& Herrmann, 2005).

When the response times for negative words were examined, it was seen that they were processed more slowly compared to positive and neutral words, another finding with considerable support in the literature. According to one view, negative stimulus may increase the amount of interference in lexical processing (Sutton \& Altarriba, 2008), making it difficult to focus on the semantic analysis. Another view maintains that negative stimulus leads to an increased workload for cognitive processing (Estes \& Adelman, 2008) by causing distraction. Also, Larsen, Mercer, Balota, and Strube (2008) argue that negative words are less frequent than positive words, and cause a novelty effect on participants, which, in turn, delays processing speed This view has a great deal of experimental support (Kissler \& Koessler, 2011; Sutton \& Altarriba, 2008). Evolutionary-based research also gives considerable support. For example, Automatic Vigilance Hypothesis predicts that negative stimuli engage attention to the extent that it slows processing speed (Estes \& Adelman, 2008).

Also, the lower frequency with which negative words are used is considered to account for the difference in processing speed. For example, Larsen, Kimberley, Mercer, and Balota (2006) maintained that studies that employed a variety of tasks, such as Lexical Decision Task, Stroop Task or Naming Task, reported longer latencies for negative words, which was taken as evidence for a correlation between frequency of use and processing speed. In Larsen et al.'s study (2008), longer latencies for negative stimuli were attributed to the novelty effect created by negative words due to their less frequent use. According to this view, negative stimuli attract our attention more quickly, however, it is more difficult to disengage or divert from the stimuli, causing a delay in processing. Our results regarding the processing of positive and negative words can be interpreted as supporting this view.

No significant differences were yielded by pair-wise comparisons of response times between the positive and neutral words in Turkish and English, and those between the negative words and neutral words in Turkish. However, a significant difference was found between the response times for negative and neutral words in English. 
These results can be explained by the tendency of neutral words to be rated higher on the concreteness scale, suggesting stronger associations in the memory (Altarriba, 2006: 234). This is assumed to account for the ease of lexical retrieval of neutral words (Sabsevitz, Medler, Seidenberg, \& Binder, 2005). In our study, the English word set includes concrete words such as Ankle, Bottle, Chair, as opposed to abstract words such as Agony, Abuse, Insult in the negative word set. This accounts for neutral words in English being processed faster than negative words. Kanske and Kotz (2007) reported a similar result. Sutton et al. (2007), similarly, reported that SpanishEnglish bilinguals processed neutral words in both languages faster than negative words. In Eilola and Havelka's (2011) study, Greek-English bilinguals processed neutral words with shorter latencies than negative words, but no significant difference was found between those for positive and neutral words.

The analysis of the accuracy rates for words in Turkish showed no significant differences between positive, negative and neutral words suggesting that the emotional content of the words have no particular effect on the accuracy of participants' responses. When the accuracy of the English words was analyzed, it was seen that positive words had higher accuracy rates compared to other words, which adds support for the Positivity Effect, already observed in the analysis of response times. Unlike response times, no significant difference was found between negative and neutral words. This result can be interpreted as the so-called Speed-Accuracy Trade-off, which maintains that participants in experimental studies may sometimes choose between speed or accuracy in their responses favoring one over the other (Bogacz, Wagenmakers, Forstmann, \& Nieuwenhuis 2010).

\section{Conclusions}

Response time data revealed superiority of positive words both in Turkish and English, in line with the literature. Accuracy rates of English words support this result. However, for Turkish words, there was no difference between the accuracy rates for positive words, and those for negative and neutral words. Also, we report faster reaction times for neutral words than negative words in English. However, this result wasn't replicated in Turkish, and similarly, there was no difference between the accuracy of neutral and negative words in either language. This study investigated processing of visually presented words in simultaneous bilinguals. Future studies may investigate whether these results are replicated when stimuli are auditorily presented, or whether the age of language acquisition and frequency of use exert influence in lexical processing.

\section{References}

Altarribba, J. (2006). Cognitive approaches to the study of emotion-laden and emotion words in monolingual and bilingual memory. In A. Pavlenko (Ed.), Bilingual Minds: Emotional Experience, Expression, and Representation (pp. 232-253) Clevedon, UK: Multilingual Matters. Retrieved from http://site.ebrary.com/lib/ekonomi/docDetail.action? docID $=10120617 \&$ p00=bilingual\%20minds 
Altarriba, J., \& Basnight-Brown, D. M. (2010). The representation of emotion vs. emotionladen words in English and Spanish in the Affective Simon Task. International Journal of Bilingualism, 15 (3), 310-328.

Anooshian, L., \& Hertel, P. (1994). Emotionality in free recall: language specificity in bilingual memory. Cognition and Emotion, 8 (6), 503-514.

Bogacz, R., Wagenmakers, E. J., Forstmann, B. U., \& Nieuwenhuis, S. (2010). The neural basis of the speed-accuracy tradeoff. Trends in Neuroscience, 33 (1), 1-16.

Bradley, M. M., \& Lang, P. J. (1999). Affective norms for English words. instruction manual and affective ratings. Retrieved from: http://www.uvm .edu/ $\sim$ pd odds / files/papers/others/1999/bradley1999a.pdf.

Brierley, B., Medford, N., Shaw, P., \& David, A. S. (2007). Emotional memory for words: separating content and context. Cognition and Emotion, 21 (3), 495-521.

Briesemeister, B. B., Kuchinke, L., \& Jacobs A. M. (2011). Discrete emotion effects on lexical decision response times. PlosONE, 6 (8), 1-9.

Chen, P., Lin, J., Chen, B., Lu, C., \& Guo, T. (2015). Processing emotional words in two languages with one brain: ERP and fMRI evidence from Chinese-English bilinguals. Cortex, 71, 34-48.

Conrad, M., Recio, G., \& Jacobs, A. M. (2011). The time course of emotion effects in first and second language processing: a cross cultural ERP study with German-Spanish bilinguals. Frontiers in Psychology, 2 (351), 1-15.

Dewaele, J. M. (2004a). The emotional force of swear words and taboo words in the speech of multilinguals. Journal of Multilingualism and Multicultural Development, 25 (2-3), 204222.

Dewaele, J. M. (2004b). Blistering barnacles! What languages do multilinguals swear in?. Estudios de Sociolinguistica, 5 (1), 83 -105.

Dewaele, J. M. (2008). The emotional weight of I love you in multilinguals' languages. Journal of Pragmatics, 40 (10), 1753-1780.

Eilola T.M., \& Havelka, J. (2011). Behavioural and physiological responses to the emotional and taboo Stroop tasks in native and non-native speakers of English. International Journal of Bilingualism, 15 (3), 353-369.

Eilola, T., Havelka, J., \& Sharma, D. (2007). Emotional activation in the first and second language. Cognition and Emotion, 21 (5), 1064-1076.

Estes, Z., \& Adelman, J. S. (2008). Automatic vigilance for negative words in lexical decision and naming: Comment on Larsen, Mercer and Balota. Emotion, 8(4), 441-444.

Ferre, P., Garcia, T., Frage, I., Sanchez-Casas, R., \& Molero, M. (2010). Memory for emotional words in bilinguals: do words have the same emotional intensity in the first and in the second language? Cognition and Emotion, 24 (5), 760-785.

Göz, İ. (2003). Yazllı Türkçe’nin kelime sıklı̆̆ı sözlüğü. Ankara: Türk Dil Kurumu Yayınları.

Harris, C. L. (2004). Bilingual speakers in the lab: psychophysiological measures of emotional reactivity. Journal of Multilingual and Multicultural Development, 25 (2), 223-247.

Harris, C. L., Ayçiçeği, A., \& Gleason, J. B. (2003). Taboo words and reprimands elicit greater autonomic reactivity in a first language than in a second language. Applied Psycholinguistics, 24 (4), $561-579$.

Harris, C. L., Gleason, J. B., \& Ayçiçeği, A. (2006). When is first language more emotional? psychophysiological evidence from bilingual speakers. In A. Pavlenko (Ed.), Bilingual minds: Emotional Experience, Expression, and Representation, (pp. 257- 283). Clevedon, UK: Multilingual Matters. http://site.ebrary.com/lib/ ekonomi/ docDetail.action? docID=10120617\&p00=bilingual\%20minds 
Jonczyk, R. (2013). Hemispheric asymmetries for emotional verbal stimuli in Polish-English bilinguals. Retrieved from http://wa.emu.edu.pl/plu/2013/files/Abstracts/Jonczyk.pdf

Jonczyk, R. (2014). Hemispheric asymmetry of emotion words in a non-native mind: A divided visual field study. Laterality, 10 (1), 1-22.

Kanske, P., \& Kotz, S. A. (2007). Concreteness in emotional words: ERP evidence from a hemifield study. Brain Research, 1148, 138-148.

Kissler, J., Herbert, C., Winkler, I., \& Junghofer, M. (2009). Emotion and attention in visual word processing - an ERP study. Biological Psychology, 80 (1), 75-83.

Kissler, J., \& Koessler, S. (2011). Emotionally positive stimuli facilitate lexical decisions - an ERP study. Biological Psychology, 86 (3), 254-264.

Kuchinke, L., Jacobs, A. M., Grubich, G., Vo, M. L., Conrad, M., \& Herrmann, M. (2005). Incidental effects of emotional valence in single word processing: an fMRI study. NeuroImage, 28 (4), 1022-1032.

Larsen, R. J., Kimberley, A., Mercer, K. A., \& Balota, D. A. (2006). Lexical characterstics of words used in emotional stroop experiments. Emotion, 6 (1), 62-72.

Larsen, R. J., Mercer, K. A., Balota, D. A., \& Strube, M. J. (2008). Not all negative words slow down lexical decision and naming speed: Importance of word arousal. Emotion, 8(4), 445452.

Nagae, S., \& Moscovitch, M. (2002). Cerebral hemispheric differences in memory of emotional and non-emotional words in normal individuals. Neuropsychologica, 40 (9), 1601-1607.

Oldfield, R. C. (1971). Oldfield handedness inventory. Neuropsychologia, 9(1),97-113.

Palazova, M., Mantwill, K., Sommer, W., \& Schacht, A. (2011). Are emotion effects in single words non-lexical? Evidence from event-related potentials. Neuropsychologia, 49 (9), 27662775.

Pavlenko, A. (2004). "Stop doing that, la komu skalaza!": Language choice and emotions in parent-child communication. Journal of Multilingual and Multicultural Development, 25 (23), $179-203$.

Pavlenko, A. (2008). Emotion and emotion-laden words in the bilingual lexicon. Bilingualism: Language and Cognition, 11(2), 147-164.

Pavlenko, A. (2012). Affective processing in bilingual speakers: disembodied cognition? International Journal of Psychology, 47 (6), $405-428$.

Sabsevitz, D. S., Medler, D. A., Seidenberg, M., \& Binder, J. R. (2005). Modulation of semantic system by word imageability. NeuroImage, 27 (1), 188-200.

Schacht, A., \& Sommer, W. (2009). Emotions in word and face processing: Early and late cortical responses. Brain and Language, 69 (3), 538-550.

Scott, G. G., O'Donnell, P. J., Leuthold, H., \& Sereno, S. C. (2009). Early emotion word processing: evidence from event-related potentials. Biological Psychology, 80 (1), 95-104.

Sutton, T. M., \& Altarriba, J. (2008). Emotion words in the mental lexicon. The Mental Lexicon, 3 (1), 29-46.

Sutton, T. M., Altarriba, J., Gianico, J. L., \& Basnight-Brown, D. M. (2007). The automatic access of emotion: emotional stroop effects in Spanish-English bilingual speakers. Cognition and Emotion, 21 (5), 1077-1090.

\section{Copyrights}

Copyright for this article is retained by the author(s), with first publication rights granted to the Journal.

This is an open-access article distributed under the terms and conditions of the Creative Commons Attribution license (CC BY-NC-ND) (http://creativecommons.org/licenses/by-nc-nd/4.0/). 